\title{
Tumorcidal Effect of Recombinant Newcastle Disease Virus
}

\author{
Katsuro Hagiwara ${ }^{*}$,, Tsuyoshi Kadosawa ${ }^{a}$ and Takaaki Nakaya ${ }^{\mathrm{b}}$ \\ ${ }^{a}$ School of Veterinary Medicine, Rakuno Gakuen University, Ebetsu, Hokkaido 069-8501 Japan \\ ${ }^{b}$ International Research Institute for Microbial Diseases, RIMD, Osaka University, 3-1 Yamadaoka, Suita, Osaka 565- \\ 0871, Japan
}

\begin{abstract}
We have generated GFP expressing recombinant Newcastle Disease Virus (rNDV-GFP) based on a live vaccine strain which contains a less pathogenic cleavage site of $\mathrm{F}$ protein leading to restricted viral growth in vivo. We evaluated the rNDV have an effect of oncolytic activity to B16 melanoma cell. The rNDV-GFP infected efficiency to B16 cells and induced cell death. Apoptosis-related genes, Bax and Caspase 3, were elevated in the infected cells. In order to evaluate the tumorcidal effect of rNDV on canine tumor cells, two canine tumors such as highly metastasizing canine osteosarcoma (HMPOS) and transitional cell carcinoma (TCC) were infected with rNDV. The rNDV induced cell death to canine tumor cells within 48 hours post infection. These results suggest that rNDV-GFP have a tumorcidal activity to B16 mouse melanoma and canine tumors in vitro.
\end{abstract}

Keywords: NDV, oncolytic, melanoma, HMPOS, TCC.

\section{INTRODUCTION}

For the treatment of tumor metastasis, various studies have previously been carried out; however, the prevention of malignant tumor metastasis is remains a difficult problem to solve. In particular, the incidence of tumor metastasis is higher in malignant tumors such as melanoma, osteosarcoma and transitional cell carcinoma. It is well known that these tumors often induce the malignant metastasis in dogs. Tumor metastasis is related to an immunity function to eradicate circulating tumor cells or tumor cells in certain regions in the organ. It is necessary to induce a cytotoxic $\mathrm{T}$ cell response against tumor cells to control metastasis. Recently, an oncolytic virus has been studied for a novel anticancer strategy $[1,2]$. Newcastle Disease Virus (NDV) has shown a tumorcidal effect on several tumors in recent years, and phase I and II clinical trials have been examined [3-5]. Several phase II trials of postoperative tumor vaccination with NDVmodified, patient-derived, live tumor cells or viral oncolysates indicated clinical benefits $[6,7]$.

NDV is a nonsegmented RNA virus of negative polarity that belongs to the paramyxovirus family. This virus is the causative agent of Newcastle disease (ND) that causes respiratory disease in birds. Interestingly, NDV, an avian paramyxovirus, replicates tumors selectively in mammals. Phase I and II trials are ongoing to evaluate NDV as an antitumor reagent in humans $[3,8,9]$. Recently, we succeeded in producing an infectious virus with the reverse genetics method from genome complementary DNA of the NDV vaccine strain (B1) [10]. This method allows us to insert a foreign gene into the NDV genome to express the foreign gene at a high rate during viral replication. In this study, by using green fluorescent protein (GFP) expressing recombinant NDV (rNDV-GFP) [11], we evaluated the cytotoxic effect of rNDV on a B16 melanoma in vitro as well as direct cell killing activity against canine tumors.

*Address correspondence to this author at the School of Veterinary Medicine, Rakuno Gakuen University, Ebetsu, Hokkaido 069-8501 Japan;

E-mail:k-hagi@rakuno.ac.jp

\section{MATERIALS AND METHODS}

\section{Rescue of Recombinant NDV from Cloned cDNA}

pNDV/B1, containing full-length cDNA of the Hitchner B1 strain was constructed (Fig. 1), and additional restriction enzyme sites (XbaI, nt 3163 to 3168 ) were created as genetic tag sequences [10]. To generate rNDV/-GFP, HEp-2 cells in a six-well plate were infected with recombinant vaccinia virus (MVA-T7) that expressed T7 RNA polymerase at a multiplicity of infection (MOI) of 1 and then transfected with each NDV-GFP full-length clone $(1 \mu \mathrm{g})$ together with the following expression plasmids: pTM1-NP (nucleoprotein) at $0.4 \mu \mathrm{g}$, pTM1-P (phosphoprotein) at $0.2 \mu \mathrm{g}$, and pTM1-L (RNAdependent RNA polymerase) at $0.2 \mu \mathrm{g}$ as described previously [10]. The transfected cells were injected into the allantoic cavities of 9- or 10-day-old embryonate chicken eggs and rNDV-GFP was harvested. The focus-forming unit (FFU) of rNDV-GFP was determined by flow cytometry analysis (Beckman Coulter Co.) using mouse B16 cells. A total of $10^{5}$ of B16 cells were infected with serial 10-fold dilutions of rNDV-GFP in 24-well plates (four wells per dilution). The infected cells were incubated for 24 hours and were harvested with $0.25 \%$ trypsin-EDTA (SIGMA). Cell suspensions were applied to flow cytometry (FCM) and the FFU/ml determined from the numbers of rNDV-GFP-infected cells.

\section{Tumor Cells}

B16 mouse melanoma cells were cultured with DMEM (Sigma) supplemented with $10 \%$ FCS. Two canine derived tumors such as Highly Metastasizing canine osteosarcoma (HMPOS) [12] and Canine transitional cell carcinoma (TCC) [13] were cultured with RPMI 1640 (Sigma) supplemented with $10 \%$ FCS. These tumor cells were cultured until logarithmic phase growth and used for the experiment.

\section{Tumorcidal Effect of rNDV on Melanoma B16 Cells and Canine Tumors}

To examine the cytotoxicity of rNDV-GFP to B16 cells, cells $\left(10^{5}\right)$ were cultured with DMEM supplemented with 


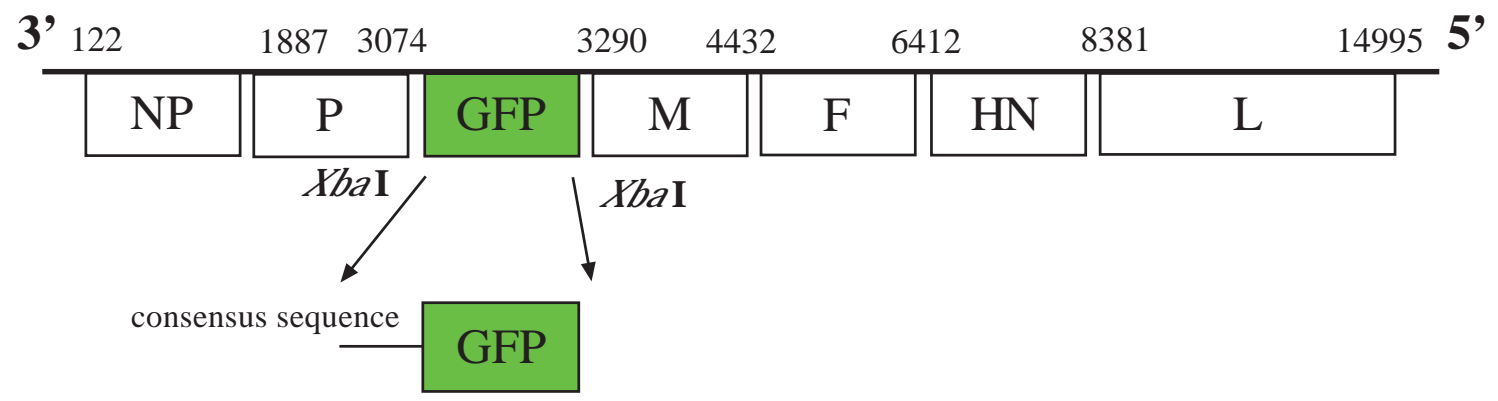

Fig. (1). Construct of rNDV-GFP genome. pNDV/B1-GFP constructs were made by inserting the GFP reading frames (ORF), respectively, into the unique $X b a \mathrm{I}$ cloning site (nt 3163) located between the $\mathrm{P}$ and $\mathrm{M}$ genes of the $\mathrm{pNDV} / \mathrm{B} 1$ clone.

$10 \%$ FCS in 24-well plates and infected with rNDV-GFP at MOI of 0.8 . The infected cells were incubated with propium iodide (50ug/ml; PI, SIGMA) at 5, 8 and 20 hours postinfection. After $10 \mathrm{~min}$ incubation with PI, the cells were washed with phosphate-buffered saline (PBS) and harvested with $0.25 \%$ trypsin-EDTA. The cells were then applied to flow cytometry (FCM). FCM analysis was performed using an argon ion laser at $488 \mathrm{~nm}$ (Beckman Coulter Co.). FITC was measured on the FL1 channel and PI on the FL2 channel, generating a log FL1 (Y-axis) versus FL2 (X-axis). Canine tumors, HMPOS and TCC, in RPMI-1640 (with 10\% FCS) were prepared at $10^{5}$ in 24 -well plates and infected with rNDV-GFP at MOI of 0.5 . The infected cells were incubated with PI $(50 \mathrm{ug} / \mathrm{ml})$ at 24 and 48 hours post-infection. As a control, primary canine fibroblast cells were also infected with rNDV-GFP. The cells were then applied to FCM as described above.

\section{Quantification of the Apoptosis-Related Gene Expression}

To verify that rNDV induced apoptosis in B16 cells, rNDV-GFP-infected cells were monitored for apoptosisrelated gene expression such as Caspase 3 and Bax by realtime (RT) PCR. Total RNA was extracted from rNDV-GFPinfected cells using Trizol reagent (Invitrogen), and cDNA was synthesized by Oligo dt (20) Primer using a reverse transcriptase kit (Roche). Absolute quantification of Caspase 3 and Bax was examined by Lightcycler 480 using a Sybergreen RT-PCR kit (Roche) according the methods described in the kit manual. Primer pairs for Caspase 3, sense 5'-ATG GGAGCAGTCAGTGGAC-3', antisense 5'-CGTACCAGA GCGAGATGACA-3' and Bax, sense 5'-GCTGATGGCAAC TTCAACTG-3', antisense 5'-GATCAGCTCGGGCACTTT AG-3', and with control primer, GAPDH sense 5'-ACCAC AGTCCATGCCATCAC-3', antisense 5'-TCCACCACCCT GTTGCTGTA-3'. Sample data were normalized with the value of GAPDH gene expression.

\section{Detection of Active Caspase 3 in B16 Infected with rNDV-GFP}

The rNDV-GFP-infected B16 cells were monitored for active Caspase 3 by FCM analysis using an APO ACTIVE 3 TM Kit (Cell Technology, Inc. CA, USA). To detect active Caspase 3, B16 ( $10^{5}$ cells) were infected with rNDV-GFP at MOI of 0.2 as described above, and then the cells undergoing apoptosis were fixed and permeabilized prior to the addition of the primary antibody which recognized the p18 subunit of cleaved Caspase 3 according the protocol of the kit. A secondary FITC-labeled goat anti-rabbit antibody was used to visualize the bound rabbit anti-Caspase 3 polyclonal antibody. FCM analysis was performed using an argon ion laser at $488 \mathrm{~nm}$. FITC was measured on the FL1 channel and a $\log$ FL1 (X-axis) versus the number of cells (Y-axis) histogram was generated.

\section{RESULTS}

To examine the cytotoxicity of rNDV-GFP to B16 cells, the cells were infected with rNDV-GFP. The GFP expression after virus infection was confirmed in B16 cells $(35 \%)$ at 5 hours post-infection (hpi) and increased up to $8 \mathrm{hpi}$ $(78 \%)$. The cytotoxicity of the infected cells was monitored by staining with propium iodide (PI) and the positive cell rate was monitored by FCM. The numbers of GFP- and PIpositive cells were few until $8 \mathrm{hpi}$, but they increased to $68 \%$ by 20 hpi (Fig. 2). To investigate the effect of the virus infection on apoptosis-related gene expression, the mRNAs from the virus infected cells were examined by RT realtimePCR analysis. The RT realtime- PCR results showed that Bax and Caspase 3 mRNA expression were significantly increased in rNDV-infected cells after 16 to 24 hpi (Fig. 3). Flow cytometry analysis also confirmed significantly the increase of active Caspase 3-positive cells at 24 hpi (Fig. 4), suggesting that rNDV induced Caspase 3-dependent apoptosis in infected cells.

Next, we examined a cytopathic effect (CPE) of rNDVGFP on two different canine tumors (HMPOS and TCC). These cells were infected with rNDV-GFP at MOI of 0.5 , and then the cell cytotoxicity was evaluated by PI staining. The infectivity of rNDV-GFP was varying in the different canine tumors. FCM analysis showed that a total of 30.5$53.9 \%$ of the cells were infected with rNDV-GFP until 48 hours post infection, and the infected cells showed a CPE as result in an increase in PI positive cells (dead cells). The rate of virus induced cell death was significantly higher in HMPOS (53.9\% of cells were dead within $24 \mathrm{~h}$ ) than that of TCC (30.5\%), but all the rNDV-GFP infected cells also reacted positively to PI within 48 hours. In contrast to the tumor cells, normal fibroblast cells did not show significant CPE by rNDV infection (Table 1).

\section{DISCUSSION}

NDV is well known to infect various cells and to induce cell death soon after infection. In this experiment, we generated GFP expressing rNDV which infected mammalian cells and induced CPE. The rNDV can infect B16 cells without viral propagation and express GFP protein in the cells. The virus leads to the death of only infected cells, without producing any infectious virus particles, since the cleavage site 


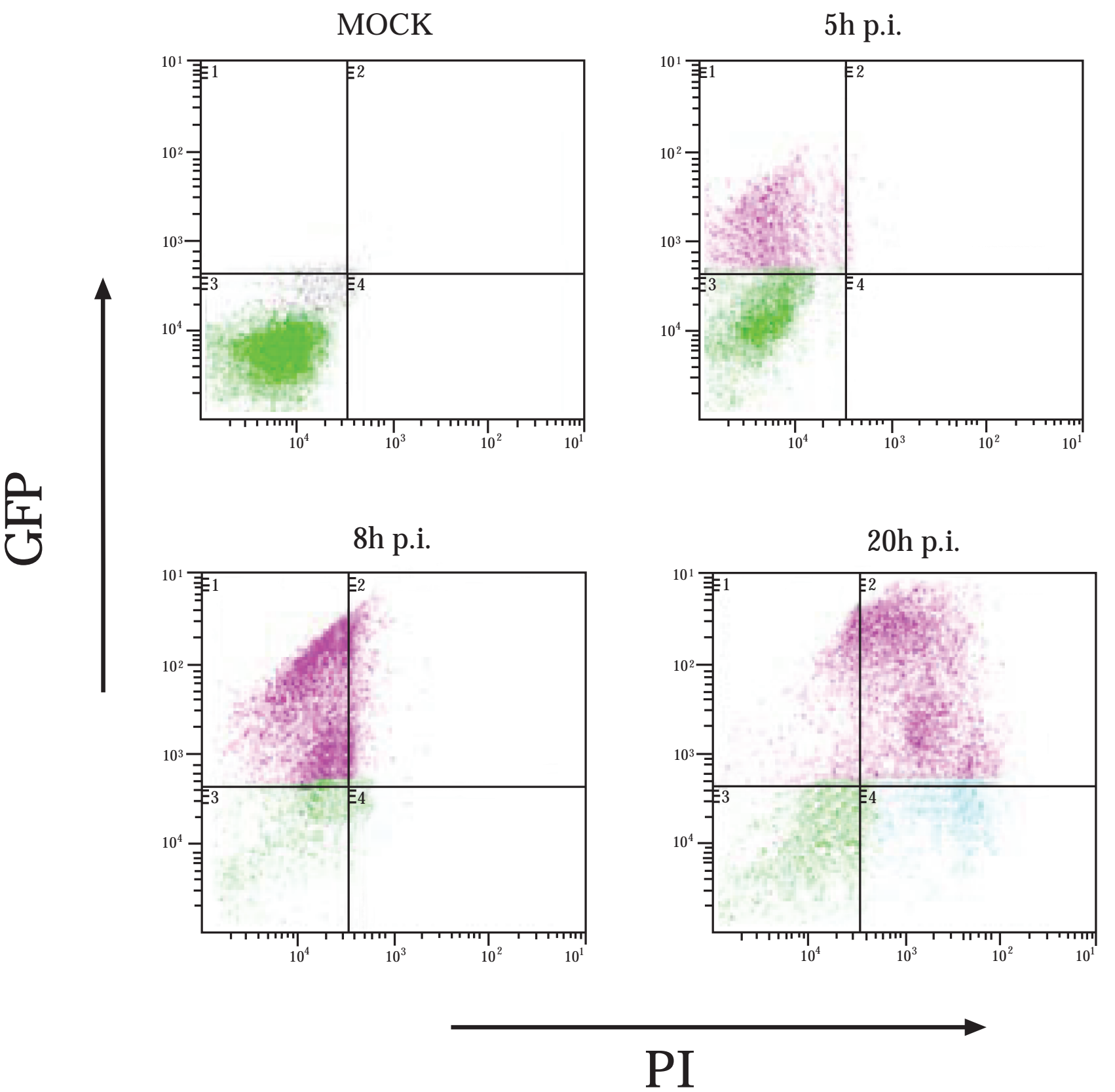

Fig. (2). Flow cytometry analysis of rNDV-infected B16 cells. Detection of rNDV-infected cells and PI-positive cells following rNDV-GFP infection. $\mathrm{Y}$ axis indicates GFP expression and $\mathrm{X}$ axis indicates PI-positive ratio.

of amino acid sequences of virus $\mathrm{F}$ protein is transformed to an attenuated virus type (G-R-Q-G/S-R $\downarrow L)$. FCM analysis indicated that more than $80 \%$ of the GFP-positive cells were PI positive at 20 hours after rNDV infection. A total of $68 \%$ of B16 were dead by rNDV infection at MOI of 0.8 . Viral oncolytic exploit the natural cytotoxicity of viruses to directly kill tumor cells, but do not show cytotoxicity to normal cells [14-16].

FCM analysis showed that virus replication (GFP expression) was detected at 5 hours after infection and dramatically increased the number of GFP- and PI-positive cells at 8 to 24 hours after rNDV-GFP infection. To investigate apoptosis contributing to virus-induced cell death, apoptosis-associated gene expressions of Caspase 3 and Bax were monitored by $\mathrm{RT}$ realtime-PCR. The central component of this process is the cascade of proteolytic enzymes called Caspase. These enzymes participate in a series of reactions that are triggered in response to pro-apoptotic signals and result in the cleavage of protein substrates, causing disassembly of the cell [17]. Caspase 3, also known as CPP-32, Apopain or Yama, is a key effector caspase in the apoptotic pathway [18]. FCM analysis disclosed that active Caspase 3-positive cells were increased at 24 hours after rNDV-GFP infection. The analysis results showed the expression of Caspase 3 and Bax genes following the increase of active Caspase 3-positive cells soon after infection. The aforementioned result clearly 
showed the induction of apoptosis to cells infected with rNDV.

Recently, it has reported that MTH-68/H, a NDV strain, was found to be able to kill a wide range of transformed cells by apoptosis and that the activation of caspase- 3 and -12 was detected in virus-infected PC12 cells, while caspase- 8 and -9 are not involved in MTH-68/H-induced apoptosis [19]. Elankumaran et al., also showed that cytotoxicity against tumor cells is due to multiple caspase-dependent pathways of apoptosis independent of interferon signaling competence [20]. These findings together with our study strongly suggest that rNDV induces tumor-specific cell death via a variety of caspase pathways.

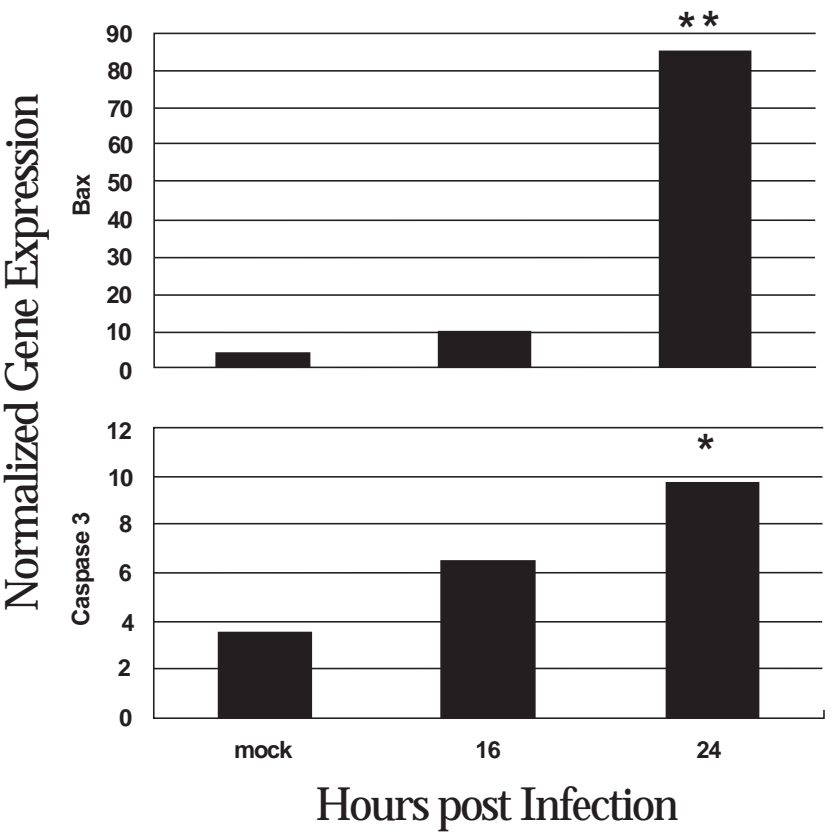

Fig. (3). Apoptosis-related gene expression in B16 cells infected with rNDV. B16 cells were infected with rNDV-GFP at MOI of 0.5. Bax (A) and Caspase 3 (B) gene expression. The statistically significant of gene expression between mock and rNDV infection were shown by asterisks $(* \mathrm{P}<0.05, * * \mathrm{P}<0.01)$.

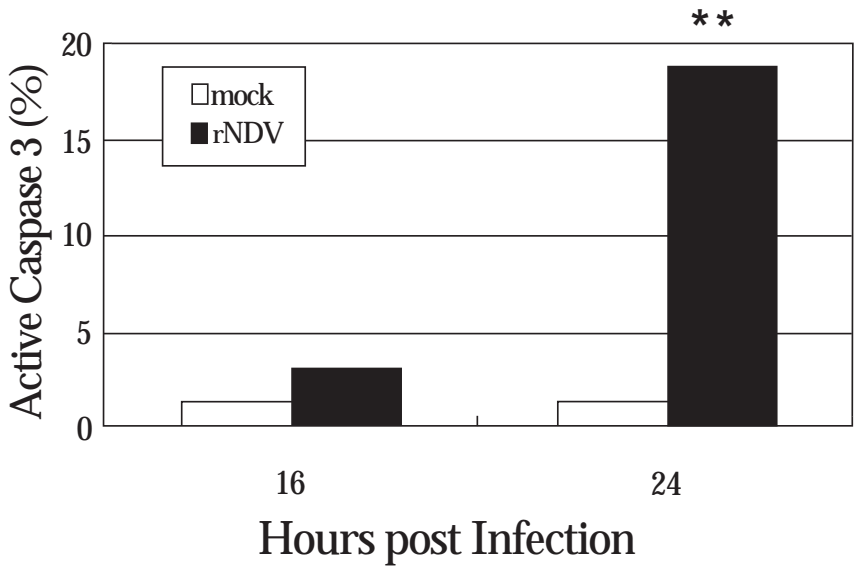

Fig. (4). Active Caspase 3-positive cells at 24 hours after rNDVGFP infection. B16 cells were infected with rNDV-GFP at MOI of 0.2. Active Caspase 3-positive cells were detected by FCM analysis. The statistically significant of Caspase 3 positive cell ratio between mock and rNDV infection were shown by asterisks $(* *$ $\mathrm{P}<0.01)$.
Newcastle disease virus affects the activation of cellmediated immunity.

In this study, the infections of rNDV-GFP clearly showed cytotoxicity to the canine derived tumors in vitro. It is conceivable that the effect of virus-induced cytotoxicity in rNDV-treated canine tumors. Osteosarcoma is one of the most malignant tumors of both man and animals. It has a high percentage of lung metastasis in its early stages. In this

Table 1. Tumorcidal Effect of rNDV-GFP on Canine Tumors

\begin{tabular}{|c|c|c|c|}
\hline & $\mathbf{2 4 h}$ & $\mathbf{4 8 h}$ & Mock \\
\hline \hline HMPOS & $53.9^{* *}$ & NT & 0.2 \\
TCC & $15.0^{*}$ & $30.5^{*}$ & 0.1 \\
Fibroblast & 4.2 & 6.5 & 0.1 \\
\hline
\end{tabular}

The average ratio of PI positive cells from three different experiments was shown. NT: not tested. The difference of PI positive ratio between tumor cells and fibroblast were statistically significant. $* \mathrm{P}<0.05, * * \mathrm{P}<0.01$.

experiment, rNDV induced a significant CPE to HMPOS within 24 hpi. It is well known that TCC often induce the malignant metastasis in dogs. Distant metastases, including regional lymph nodes, occur in approximately half of all bladder TCCs and one third of urethral TCCs [21, 22]. The effect of cytotoxicity of rNDV was $30.5 \%$ in TCC. The reduction of infectivity and cytotoxicity in this tumor cells is not clear, but the difference in results may be explained by the varying infectivity and virus replication in each canine tumor cells. This point will have to study in detail in future.

In conclusion, rNDV-GFP infects mouse and canine tumor cells. The results showed rNDV has a tumorcidal activity to B16 mouse melanoma and canine tumors (HMPOS and TCC) in vitro.

\section{ACKNOWLEDGEMENTS}

This study was partly supported by a grant for furtherance (adoption No. 7) at Rakuno Gakuen University and a grant-in-aid for the High Technological Research Center (Rakuno Gakuen University) from the Ministry of Education, Science, Sports and Culture of Japan.

\section{REFERENCES}

[1] Aghi M, Martuza RL. Oncolytic viral therapies - the clinical experience. Oncogene 2005; 24(52): 7802-7816.

[2] Woo Y, Adusumilli PS, Fong Y. Advances in oncolytic viral therapy. Curr Opin Investig Drugs 2006; 7(6): 549-559.

[3] Freeman AI, Zakay-Rones Z, Gomori JM, et al. Phase I/II trial of intravenous NDV-HUJ oncolytic virus in recurrent glioblastoma multiforme. Mol Ther 2006; 13(1): 221-228.

[4] Sinkovics JG, Horvath JC. Newcastle disease virus (NDV): brief history of its oncolytic strains. J Clin Virol 2000; 16(1): 1-15.

[5] Schirrmacher V, Bai L, Umansky V, Yu L, Xing Y, Qian Z. Newcastle disease virus activates macrophages for anti-tumor activity. Int J Oncol 2000; 16(2): 363-373.

[6] Schirrmacher V, Beutner U, Bucur M, Umansky V, Rocha M, von Hoegen P. Loss of endogenous mouse mammary tumor virus superantigen increases tumor resistance. J Immunol 1998; 161(2): 563-570.

[7] Cassel WA, Murray DR. A ten-year follow-up on stage II malignant melanoma patients treated postsurgically with Newcastle disease virus oncolysate. Med Oncol Tumor Pharmacother 1992; 9(4): 169-171. 
[8] Laurie SA, Bell JC, Atkins HL, et al. A phase 1 clinical study of intravenous administration of PV701, an oncolytic virus, using two-step desensitization. Clin Cancer Res 2006; 12(8): 2555-2562.

[9] Schirrmacher V. Clinical trials of antitumor vaccination with an autologous tumor cell vaccine modified by virus infection: improvement of patient survival based on improved antitumor immune memory. Cancer Immunol Immunother 2005; 54(6): 587598.

[10] Nakaya T, Cros J, Park MS, et al. Recombinant Newcastle disease virus as a vaccine vector. J Virol 2001; 75(23): 11868-11873.

[11] Park MS, Garcia-Sastre A, Cros JF, Basler CF, Palese P. Newcastle disease virus $\mathrm{V}$ protein is a determinant of host range restriction. $\mathrm{J}$ Virol 2003; 77(17): 9522-9532.

[12] Kadosawa T, Nozaki K, Sasaki N, Takeuchi A. Establishment and characterization of a new cell line from a canine osteosarcoma. J Vet Med Sci 1994; 56(6): 1167-1169.

[13] Takagi S, Kitamura T, Hosaka Y, et al. Molecular cloning of canine membrane-anchored inhibitor of matrix metalloproteinase, RECK. J Vet Med Sci 2005; 67(4): 385-391.

[14] Fiola C, Peeters B, Fournier P, Arnold A, Bucur M, Schirrmacher $\mathrm{V}$. Tumor selective replication of Newcastle disease virus: association with defects of tumor cells in antiviral defence. Int $\mathrm{J}$ Cancer 2006; 119(2): 328-338.
[15] Reichard KW, Lorence RM, Cascino CJ, et al. Newcastle disease virus selectively kills human tumor cells. J Surg Res 1992; 52(5): 448-453.

[16] Washburn B, Schirrmacher V. Human tumor cell infection by Newcastle Disease Virus leads to upregulation of HLA and cell adhesion molecules and to induction of interferons, chemokines and finally apoptosis. Int J Oncol 2002; 21(1): 85-93.

[17] Slee EA, Adrain C, Martin SJ. Serial killers: ordering caspase activation events in apoptosis. Cell Death Differ 1999; 6(11): 10671074.

[18] Alnemri ES, Livingston DJ, Nicholson DW, et al. Human ICE/CED-3 protease nomenclature. Cell 1996; 87(2): 171.

[19] Fabian Z, Csatary CM, Szeberenyi J, Csatary LK. p53-independent endoplasmic reticulum stress-mediated cytotoxicity of a Newcastle disease virus strain in tumor cell lines. J Virol 2007; 81(6): 28172830.

[20] Elankumaran S, Rockemann D, Samal SK. Newcastle disease virus exerts oncolysis by both intrinsic and extrinsic caspase-dependent pathways of cell death. J Virol 2006: 7522-7534.

[21] Norris AM, Laing EJ, Valli VE, et al. Canine bladder and urethral tumors: a retrospective study of 115 cases (1980-1985). J Vet Intern Med 1992; 6(3): 145-153.

[22] Klausner JSC, D. D., ed. Neoplasms of the urinary tract. Baltimore: Williams and Wilkins. 1995. 